\title{
A method for converting the Ansys and Ls-dyna finite element model into SASSI code
}

\author{
W. T. Li \& Q. J. Chen \\ State Key Laboratory of Disaster Reduction in Civil Engineering, \\ Tongji University, China
}

\begin{abstract}
In most national nuclear seismic codes, seismic analysis on the basis of dynamic soil-structure interaction is a basic requirement. However, after decades of intensive study, a certain gap between the research activities and practical activities remains. This is due, to a large extent, to the different computational concepts between code SASSI and the other general codes, such as Ls-dyna, Ansys and Abaqus. In this paper, a method for converting the Ansys/Ls-dyna finite element model into SASSI is developed, and the corresponding code is programmed in Matrix Laboratory (MATLAB) and Ansys Parametric Language (APDL). Through this method, firstly, the input files of every SASSI module are automatically generated according to the Ansys or Ls-dyna finite element model. Next, the SASSI code is called, and its modules are conducted sequentially until the full analysis is completed. The structural response results that are required by users are outputted and saved in the expected format. In this paper, after introducing the method step-by-step, the dynamic soil-structure interaction simulations of a pile-high-rise structure and a nuclear factory are, respectively, conducted using the Ls-dyna and SASSI programs. The comparison results prove the feasibility of the method produced in this paper.
\end{abstract}

Keywords: soil-structure interaction (SSI), seismic analysis, finite element, Ansys, Ls-dyna, SASSI, nuclear structure, program.

\section{Introduction}

The 21 st century has experienced a high incidence of earthquakes, with both the frequency and magnitude increasing sharply. Seismic analysis on the basis of dynamic structure-foundation-soil interaction is a basic requirement in most 
national nuclear seismic codes. Many papers [1-5], have also confirmed the important influence of soil structure interaction on the design of nuclear facilities.

The research on soil-structure interaction (SSI) can be traced to the late 19th century (Wang et al. [6]). The study of SSI began with Reissner's 1936 proposal of a vibrational foundation theory (Reissner [7]). In the early work, all of the approximate models had various handicaps that led to considerable misestimations of the energy passing away from the volume. A breakthrough was achieved when Lysmer and Waas [8] developed the special boundary conditions for the layered media (called "the transmitting boundary"). To date, the SSI specialists use SASSI as an industrial standard, and the comparative tests versus the field results prove its quality. Many papers [9-14], have confirmed the important influence of SSI.

Currently, many research studies are focused on the seismic safety of nuclear structures. The Nuclear Power Engineering Corporation (NUPEC) has conducted a series of research projects on SSI under the entrustment of the MITI (Ministry of International Trade and Industry Japan) to ensure the adequacy of the seismic design methodologies used in nuclear power plant (NPP) reactor building design Kitada et al. [15]. Many other papers [16, 17], have researched the seismic environment of nuclear facilities.

However, after decades of intensive studies of the soil-structure interaction, there is still a certain gap between the SSI specialists, who usually use SASSItype codes, and the civil engineers, who usually use the more general programs. The authors believe one of the critical reasons for this divide is the different modelling concept in code SASSI versus the general codes, such as Abaqus, Lsdyna and Ansys. It results in the situation that "neither current SASSI, nor general codes can repeat the results of the "other party' in order to further demonstrate step by step the nature of the difference in the analytical results" (Tyapin [18]).

This paper produces a method for converting an Ansys or Ls-dyna FE model into SASSI. Through this approach, the input files of the SASSI modules can be automatically generated according to the Ansys or Ls-dyna FE model. Next, the SASSI program is called, and its modules are conducted sequentially. Finally, the structural response results requested by users are outputted and saved in the expected format. Using the method described in this paper, the SASSI simulation results can be obtained directly from the Ansys or Ls-dyna FE model. The corresponding code $\mathrm{Li}$ and Chen [19] of this method is programmed in Matrix Laboratory (MATLAB) and Ansys Parametric Language (APDL). Notably, before and after converting, the parameters, such as the node number, node coordinates, element number, element nodes, material number, and element section proprieties, are all the same; this approach is convenient for comparing the results that were separately obtained from those two part programs.

The outline of this paper is as follows. First, the method for converting the Ansys (or Ls-dyna) FE model into SASSI is introduced. Next, the SSI dynamic simulation results of a pile-high-rise structure that are respectively calculated by Ansys (Ls-dyna) and SASSI are given. Subsequently, the SSI dynamic simulation results of a nuclear factory respectively calculated by Ansys (Ls- 
dyna) and SASSI are given. Through the two examples, the reasonability of the converting method produced in this paper is verified.

\section{Method for converting the Ansys (or Ls-dyna) FE model into SASSI}

\subsection{Introduction}

Ansys, as a widely used engineering software package, is familiar to engineers. Ansys Ls-dyna combines the Ls-dyna explicit finite element program with the pre- and post-processing capabilities of the Ansys program.

SASSI, which is specialised for SSI analysis and depends on the substructuring subtraction method, consists of a number of interrelated computer program modules. It can be used to solve a range of dynamic SSI problems. In SASSI, the site consists of semi-infinite elastic or viscoelastic horizontal layers on a rigid base or a semi-infinite elastic or viscoelastic halfspace. The seismic environment may consist of an arbitrary three-dimensional superposition of inclined body waves and surface waves or incoherent ground motion. The basic methods of analysis are called the flexible volume and subtraction methods. These methods are formulated in the frequency domain using the complex response method and the finite element technique. The complete soil-structure system, as shown in fig. 1(a), is partitioned into two substructures, namely, the foundation and the structure, as shown in fig. 1(b) and fig. 1(c), respectively. Interaction between the structure and the foundation occurs at all basement nodes.

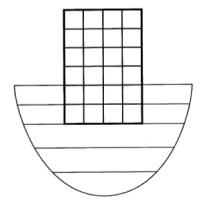

(a) Total system

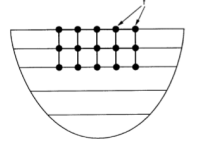

(b) Foundation

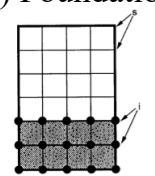

(c) Structure

Figure 1: Substructuring in the flexible volume method.

Our code for converting an Ansys (or Ls-dyna) FE model into SASSI is written in Ansys Parametric Language (APDL) and Matrix Laboratory 
(MATLAB). APDL is a scripting language that can be used to automate Ansys common tasks or build the Ansys FE model in terms of parameters. MATLAB is a multi-paradigm numerical computing environment and programming language. MATLAB can host the ActiveX control, i.e., it can control the extra-program from MATLAB using ActiveX. In our code, we use the ActiveX to call SASSI and use the APDL language to obtain the geometry or properties parameters from the Ansys or Ls-dyna FE model.

\subsection{Step-by-step approach}

The step-by-step approach of the method for converting the Ansys (or Ls-dyna) FE model into SASSI is as follows.

(1) Building the Ansys FE model. The Ansys FE model could be built in a variety of possible ways, such as GUI, APDL, or being imported from other software.

(2) Obtaining the geometric information (e.g. node number, node coordinates, element number, element nodes), material properties (e.g. material number, Poisson's ratio, elastic modulus, shear modulus), and element section proprieties from the Ansys (or Ls-dyna) FE model through APDL. This information will be written into the input file of the HOUSE module in SASSI through MATLAB.

(3) Defining the z-coordinate of the ground level and obtaining information of the excavated soil volume from the Ansys/Ls-dyna FE model through APDL. In SASSI, all nodes below this defined z-coordinate are assumed to be connected to the ground unless interaction nodes are specified. In this step, soil node information (e.g. node number, node coordinates), soil element information (e.g. element number, element nodes) and the soil layers that belong to each special soil element are obtained from the Ansys/Ls-dyna model through APDL. This information will be written into the input file of the SITE module in SASSI through MATLAB.

(4) Defining the soil layer proprieties (e.g. soil layer thickness, unit weight, Swave velocity, P-wave velocity, S-wave associated damping ratio and $\mathrm{P}$-wave associated damping ratio). This information will be written into the input file of the SITE module in SASSI through MATLAB.

(5) Selecting the file that stores the acceleration time history of the control motion. The data in the file will be written into Tape 7 of SASSI through MATLAB in the required format (8F9.6). The acceleration time history will be used in the later seismic analysis.

(6) Defining DT (time step) and NFFT (number of points to be used in the Fourier transform) for the selected time history of the control motion, and defining the frequency numbers that must be positive nonzero integer numbers. SASSI will compute the frequency step as $D F=\frac{1}{(\mathrm{NFFT} \times \mathrm{DT})}$ and will automatically reorder the input frequency numbers in ascending order; SASSI will stop if two or more equal-frequency numbers are detected. Frequencies $f_{i}$ for which solutions are obtained, are defined as $f_{i}=N F R(i) \times \mathrm{DF}$. The highest frequency of analysis is equal to the highest frequency number multiplied by the 
frequency step. This information will be written into the input files of the SITE and MOTION modules in SASSI through MATLAB.

(7) Defining the seismic environment. The seismic environment may be assumed to consist of one single wave type or several wave types of P-waves, $\mathrm{SV}$-waves, SH-waves, R-waves, or L-waves. The ratio of participation of each wave type can be given in this step. The incident angle of waves can also be defined. The number of layers generated to simulate the halfspace must also be defined. This information will be written into the input file of the SITE module in SASSI through MATLAB.

(8) Specifying the parameters for the response spectral analysis and defining the output control parameters. In this step, damping values for the response spectral analysis, the total duration of control motion, the number of nodal points or elements where the outputs are required, and the required output responses will be defined. For seismic analysis, the optional output responses are the transfer function, the response spectra, the nodal acceleration time history, the nodal velocity time history, the nodal displacement time history, the elemental stress time history, the elemental forces time history, and the elemental moment time history. This control parameter information will be written into the input file of the MOTION and STRESS modules in SASSI through MATLAB.

(9) Calling the SASSI code to compute and save the response results in the specified format. In this step, the SITE, POINT, HOUSE, ANALYS, MOTION and STRESS modules in SASSI will be called sequentially and run automatically. The input files that are generated in previous steps will be used in this step. After all of the modules are computed, the specified structural responses data will be outputted and saved in the expected format.

\subsection{Discussion}

The acceptable accuracy level and reasonable computational time address the most important engineering requirements. The emergence of the numerical method promotes the SSI study to a great extent. Among all the numerical methods, the finite element method (FEM) is a brilliant one. Many excellent general codes, such as Abaqus, Ls-dyna and Ansys, are developed and widely used today. These general codes provide solution of almost all types of analysis in structural mechanics. But as for the SSI problems, the code SASSI overcomes some shortcomings of the general codes. The main shortcomings of the general codes are the boundary conditions, and damping, the usually time-consuming running process.

SASSI highly improves the computation accuracy and saves the computational time of SSI problems. Up to now, the SSI specialists use SASSI as an industrial standard, and the comparative tests versus the field results prove its quality. However, unlike the general codes that use relative detailed models, SASSI models are mostly simplified to hybrid finite element model and stick model. To the authors' knowledge, this is mostly because of the restriction of SASSI's pre-processor. Thus, SASSI users' rich experience and knowledge is vital. Also, the responses in a special position of structures are often not 
outputted. This is usually not sufficient for the increasingly complex structures nowadays.

In SSI problem, the boundary conditions are important. The ideal boundary conditions should avoid the reflection of the waves into the finite soil volume. In general codes, the SSI effect considerably depends on the finite geometry of the soil. The reflection waves are hardly estimate on the artificially boundaries. However, unlike general codes, in the SASSI code, the transmitting boundaries in the frequency domain are used. It overcomes the boundary problems in general codes.

Another attractive feature of the general codes is that they are easy to modeling and implement. The great preprocessor and postprocessor of them allow the detailed simulation of problems and the detailed visualization of calculation results. However, all of this are harder in SASSI. If a SASSI model can be associated with a general code one, the numbers of nodes and elements can be easier obtained.

The general codes provide a wide range of simulation options. However, SASSI can only be used to solve the dynamic SSI problems. Thus, in most engineering design processes, a general code model is usually built. This is also a reason why engineers are more likely to use the general codes to solve SSI problems. If the SASSI model can be directly transferred from a general code model, the professional SSI analysis code SASSI maybe will more common in engineering design process.

The method proposed in this paper build a bridge between the general codes and the SSI professional code SASSI. It helps to narrow the gap between SSI specialists and civil engineers.

\section{Example 1: dynamic SSI analysis of a pile-high-rise structure}

\subsection{Problem definition and the FE models}

The structure under investigation is a square reinforced concrete frame structure. The total height of the structure is $60 \mathrm{~m}$; the width (square) of the structure is 24 $\mathrm{m}$; the number of stories is 20 . The center-to-center distance between two columns is 6 . The length of piles is $24 \mathrm{~m}$; the sectional diameter of piles is 0.35 $\mathrm{m}$. The center-to-center distance between two piles is $6 \mathrm{~m}$. The underlying soil consists of 10 layers. The equivalent shear wave velocity of soil is $361 \mathrm{~m} / \mathrm{s}$. The nonlinear phase of the soil in a strong shock is considered by the equivalent linearization method, using SHAKE.

In the Ansys/Ls-dyna FE model, the total number of elements in the Ansys or Ls-dyna model is 1,271,193. The Ansys finite element model is depicted in fig. 2. The SASSI FE model, which is directly converted from the Ansys FE model, is shown in fig. 3 . 


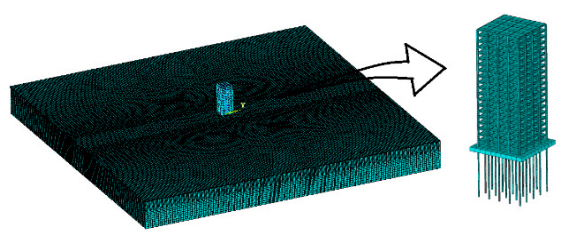

Figure 2: Ansys FE model of the high-rise structure.

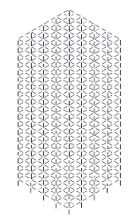

Figure 3: SASSI FE model of the high-rise structure.

\subsection{Comparison of the structural time-history acceleration responses between Ls-dyna and SASSI}

In this section, the time-history responses of the soil-structure coupled model are conducted by Ls-dyna and SASSI.

The frequently used seismic record El Centro is selected as the induced shaking wave. The amplitude of the wave is adjusted to $0.30 \mathrm{~g}$. The record is considered as vertically incident $S$ waves. The acceleration time history, Fourier spectrum, and record data are shown in fig. 4. The structural roof acceleration responses that are calculated by program Ls-dyna and SASSI are plotted in fig. 5 .
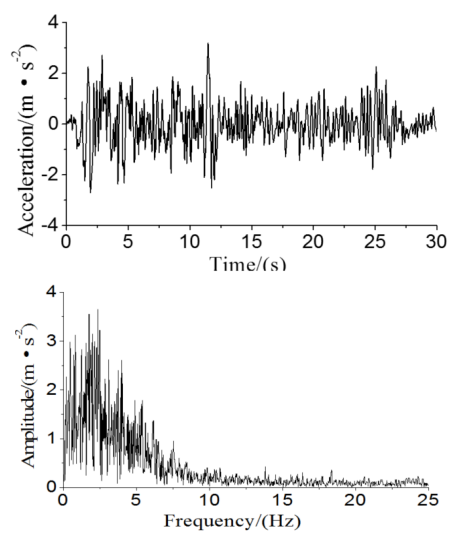

Figure 4: Seismic wave data (name: El Centro; record type: measured ground wave; location: Imperial Valley; data: 1940-05-18). 


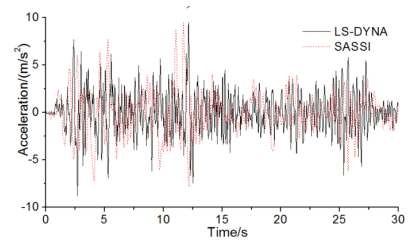

Figure 5: Structural roof acceleration histories calculated by Ls-dyna and SASSI.

\section{Example 2: dynamic SSI analysis of a nuclear factory}

\subsection{The Ls-dyna FE model and the SASSI FE model}

The factory structure is resting on slightly to moderately weathered rock. The aboveground structure is the steel frame-bent structure, and the underground structure is the reinforced concrete frame shear wall structure. The excitation load is the vertically incident shaking wave that produces motion on the rigid bedrock surface along the long-axis of the factory building.

In the Ls-dyna FE model, the structural beams and columns are simulated by beam 161, the floors are simulated by shell163, and the soil is simulated by solid164. The FE model is shown in fig. 6 .

In the SASSI FE model, the structural beams and columns are simulated by 3$\mathrm{D}$ beam elements, the floors are simulated by three- or four-node quadrilateral thin shell elements, and the excavation soil is simulated by 3-D eight-node solid elements. The FE model of the factory structure is shown in fig. 7. The FE model of the excavation soil is shown in fig. 8 .

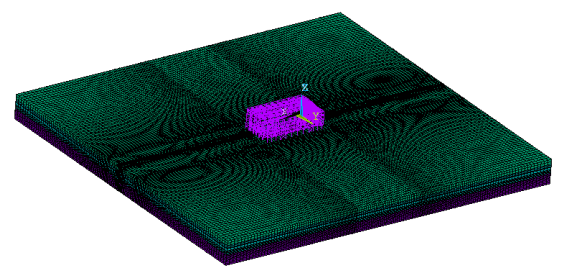

Figure 6: Ls-dyna FE model of the nuclear factory structure.

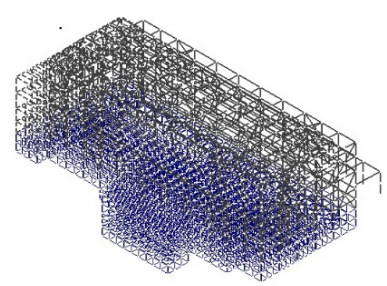

Figure 7: $\quad$ SASSI FE model of the nuclear factory structure. 


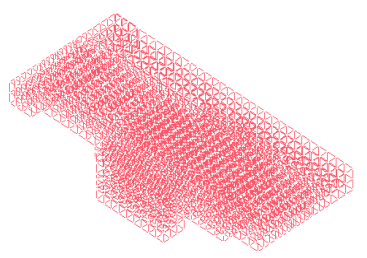

Figure 8: $\quad$ SASSI FE model of the excavation soil.

The shear wave velocity of the underlying soil varies from $119 \mathrm{~m} / \mathrm{s}$ (on the ground surface at the depth of $0 \mathrm{~m}$ ) to $532 \mathrm{~m} / \mathrm{s}$ (on the top of bedrock at the depth of $100 \mathrm{~m}$ ). The equivalent soil shear wave velocity from the ground surface to the top of bedrock is $355 \mathrm{~m} / \mathrm{s}$.

The acceleration time history of the vertically incident shaking that produces motion on the rigid bedrock surface (depth=100 m) along the structural long-axis is shown in fig. 9.

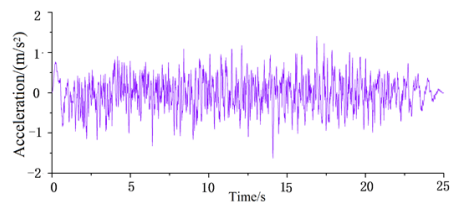

Figure 9: The acceleration time history of the shaking wave.

\subsection{Comparison of the structural time-history responses between Ls-dyna and SASSI}

The structural acceleration time-history responses at the top corner of the factory building that are calculated using Ls-dyna and SASSI are shown in figs 10 and 11 , respectively.

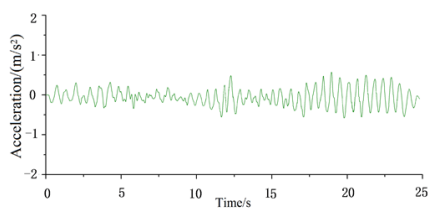

Figure 10: Structural acceleration time-history response at the top corner of the factory building calculated by Ls-dyna.

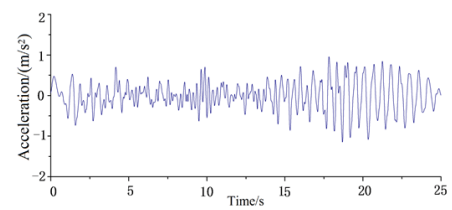

Figure 11: Structural acceleration time-history response at the top corner of the factory building calculated by SASSI. 


\section{Discussion of the two examples}

We conducted two examples to test how much the implementation of the proposed method is reasonable. The structure in one example is a pile-high-rise structure and in the other example is a nuclear factory.

In the example of the pile-high-rise structure, we can see that the acceleration amplitude calculated by Ls-dyna and SASSI are very close, both nearly $9.4 \mathrm{~m} / \mathrm{s}^{2}$ (see fig. 5). Moreover, the time that the maximum acceleration occurred in the two approaches are very close, which are $11.74 \mathrm{~s}$ and $12.04 \mathrm{~s}$, respectively.

We defined the transfer function $Q$ as $Q=a b s\left\{\frac{X_{s g}}{X_{0}}\right\}$. Where $X_{s g}$ is the amplitude of the structural horizontal displacement response; $X_{0}$ is the amplitude of the input shaking on the surface of bedrock. The transfer functions $Q$ at the structural basement are plotted in fig. 12. We can see that the first main frequency that calculated by the two approaches are very close. The match of the second main frequencies is also acceptable. The amplitude value is different at this point but the accordingly frequency value is very close. There is a third peak point in the SASSI result at the frequency of approximately $7.5 \mathrm{~Hz}$, but the third peak is missing in the Ansys result. In high frequencies, the transfer function calculated by SASSI is lower than that calculated by Ansys.

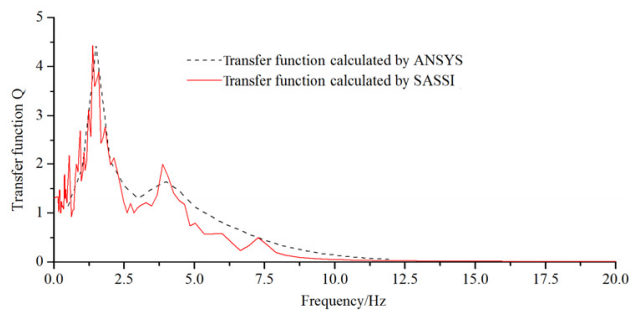

Figure 12: Transfer function $Q$ at the base of the pile-high-rise structure by Ansys and SASSI.

In the example of the nuclear factory, the acceleration amplitude calculated by Ls-dyna (see fig. 10) is $0.613 \mathrm{~m} / \mathrm{s}^{2}$ occurred at $18.84 \mathrm{~s}$ while the acceleration amplitude calculated by SASSI (see fig. 11) is $0.987 \mathrm{~m} / \mathrm{s}^{2}$ occurred at $17.82 \mathrm{~s}$. Thus, the structural acceleration response calculated by SASSI is larger than the response calculated by Ls-dyna. The percentage difference between the two acceleration amplitudes with respect to the SASSI result is $37.89 \%$, which is large but is still supposed acceptable in the engineering. The times that the maximum acceleration occurred in the two approaches are similar. The dominant periods of the acceleration responses calculated by the two approaches (Ls-dyna and SASSI) are both approximately $0.6 \mathrm{~s}$.

Beyond on the two examples, taking account into the theoretical difference between the two programs, we think the simulation results of the two programs Ls-dyna and SASSI are well matched. Thus, we deduced that the SASSI model 
which is directly transferred from the Ls-dyna model is reasonable. The method proposed in this paper is practicable.

\section{Conclusion}

This paper produced a method for converting Ansys (or Ls-dyna) finite element model into SASSI. The code of the method is programmed in Matrix Laboratory (MATLAB) and Ansys Parametric Language (APDL). Through this method, the input files of every SASSI module can be automatically generated according to the Ansys or Ls-dyna finite element model, and the analysis produces of code SASSI can be automatically conducted. To clearly illustrate the method steps and verify the converting method, dynamic soil-structure interaction simulations of a pile-high-rise structure and a nuclear factory are conducted. The simulation results in the two examples prove the reasonability of the converting method in this paper.

Before and after conversion, the parameters, such as node number, node coordinates, element number, element nodes, material number, and element section proprieties, in Ansys (Ls-dyna) FE model and SASSI FE model are all the same. Thus, this approach is convenient for comparing the results obtained from the Ansys (Ls-dyna) and SASSI programs.

What's more, the method developed in this paper can be easily applied into the converting action from the FE model of other finite element software, such as Abaqus, to the SASSI FE model. For Abaqus, the code can be written in the Python and MATLAB languages.

\section{Acknowledgements}

This work is supported by the National Science and Technology Pillar Program of China (Grant no. 2015BAK17B04). This support is gratefully acknowledged.

\section{References}

[1] Little, R. R. \& Scavuzzo, R. J., Vertical soil-structure interaction of nuclear power plants subjected to seismic excitation. Nuclear Engineering and Design, 24(2), pp. 203-213, 1973.

[2] Kamagata, S. \& Takewaki, I., Occurrence mechanism of recent large earthquake ground motions at nuclear power plant sites in Japan under soil-structure interaction. Earthquake and Structures, 4(5), pp. 557-585, 2013.

[3] Matthees, W. \& Magiera, G., A sensitivity study of seismic structure-soilstructure interaction problems for nuclear power plants. Nuclear Engineering and Design, 73(3), pp. 343-363, 1982.

[4] Roy, C., Bolourchi, S. \& Eggers, D., Significance of structure-soilstructure interaction for closely spaced structures. Nuclear Engineering and Design, 2015. 
[5] Bhaumik, L. \& Raychowdhury, P., Seismic response of nuclear reactor buildings incorporating nonlinear soil-structure interaction. Nuclear Engineering and Design, 2013.

[6] Wang, H., Lou, M., Chen, X. \& Zhai, Y., Structure-soil-structure interaction between underground structure and ground structure. Soil Dynamics and Earthquake Engineering, 54, pp. 31-38, 2013.

[7] Reissner, E., Stationäre, axialsymmetrische, durch eine schüttelnde Masse erregte Schwingungen eines homogenen elastischen Halbraumes. Archive of Applied Mechanics, 7(6), pp. 381-396, 1936.

[8] Lysmer, J. \& Waas, G., Shear waves in plane infinite structures. Journal of Engineering Mechanics, 1972.

[9] Uz, M. E. \& Hadi, M. N., Seismic history analysis of asymmetrical adjacent buildings with soil-structure interaction consideration. WIT Transaction on the built environment, 120, pp. 225, 2011.

[10] Sapountzakis, E. J. \& Kampitsis, A. E., The nonlinear vibrations of soilpile systems under seismic ground motion. WIT/Earthquake Ground Motion: Input Definition for Aseismic Design, pp. 163, 2014.

[11] Sapountzakis, E. J. \& Kampitsis, A. E., Nonlinear vibrations of piles in viscoelastic foundations. WIT Transaction on the built environment, 120, pp. 151, 2011.

[12] Favvata, M. J., Key issues regarding interaction of adjacent RC structures. WIT/Earthquake Resistant Engineering Structures X, 152, pp. 3, 2015.

[13] Chen, Q. \& Li, W., Effects of a Group of High-Rise Structures on Ground Motions under Seismic Excitation. Shock and Vibration, 501, pp. 821750, 2015.

[14] Oliveto, G. \& Brebbia, C. A., ERES 99, Earthquake resistant engineering structures II, WIT Press: Ashurst, 1999.

[15] Kitada, Y., Hirotani, T. \& Iguchi, M., Models test on dynamic structurestructure interaction of nuclear power plant buildings. Nuclear Engineering and Design, 192(2-3), pp. 205-216, 1999.

[16] Palumbo, P., Gramiccia, L., Cardellicchio, S., Faccioli, E. \& Villani, M., Garigliano nuclear power plant: seismic evaluation of the turbine building. WIT/Earthquake Resistant Engineering Structures VIII, 8, pp. 207, 2011.

[17] Dundulis, G. \& Uspuras, E., Seismic analysis of the Ignalina NPP downcomers pipes. WIT/Earthquake Resistant Engineering Structures VII, 104, pp. 459, 2009.

[18] Tyapin, A., The frequency-dependent elements in the code SASSI: A bridge between civil engineers and the soil-structure interaction specialists. Nuclear Engineering and Design. 237(12-13), pp. 1300-1306, 2007

[19] Li, W. \& Chen, Q., Fast preprocessor code of substructure analysis system SASSI 2010 (patent, in Chinese), 2015. 